\title{
Barreiras à implementação da remediação ambiental no Brasil: aspectos gerais relacionados a minas de urânio
}

\author{
Constraints for implementing environmental remediation in Brazil: political and regulatory aspects \\ related to uranium mines
}

Barreras para la implementación de la remediación ambiental en Brasil: aspectos generales relacionados con las minas de uranio

Recebido: 01/12/2020 | Revisado: 10/12/2020 | Aceito: 06/01/2021 | Publicado: 07/01/2021

\author{
Darci Geraldo Júnior \\ ORCID: https://orcid.org/0000-0002-3346-259X \\ Universidade Federal de Alfenas, Brasil \\ E-mail: darcigeraldojunior@gmail.com \\ Luis Felype Correa Paschoalin \\ ORCID: https://orcid.org/0000-0002-2637-4460 \\ Universidade Federal de Alfenas, Brasil \\ E-mail: felypeee@live.com \\ Alexandre Pereira de Oliveira \\ ORCID: https://orcid.org/0000-0002-0737-2339 \\ Comissão Nacional de Energia de Nuclear, Brasil \\ E-mail: apolivei@cnen.gov.br \\ Luciana Botezelli \\ ORCID: https://orcid.org/0000-0002-5916-0442 \\ Universidade Federal de Alfenas, Brasil \\ E-mail: luciana.botezelli@gmail.com
}

\begin{abstract}
Resumo
Remediação ambiental e descomissionamento são tendências para o desenvolvimento tecnológico, merecendo atenção particular. Este artigo visa identificar barreiras que podem retardar o descomissionamento e remediação ambiental de instalações de mineração e beneficiamento de urânio, identificando possíveis soluções. Assim, uma pesquisa em sites e bibliografias específicas foi conduzida, tendo como guia a seção 3 do Projeto CIDER. Analisando a informação reunida, o Brasil não possui uma base legal adequada para guiar a remediação ambiental de instalações de mineração e beneficiamento de urânio. Portanto, é necessário que o Brasil estabeleça uma política nacional específica, que promova seus objetivos de forma clara e transparente através todo o ciclo de vida de projetos de remediação ambiental.
\end{abstract}

Palavras-chave: Descomissionamento; Legislação; Mineração.

\begin{abstract}
Environmental remediation and decommissioning are trending topics to tecnological development, deserving particular attention. This paper consisted in identify constraints that may delay the decommissioning and environmental remediation of uranium mining and milling facilities, identifying possible sollutions. To do so, a search on specific websites and bibliographies was conducted, with the guidance of section 3 of the CIDER Project. Analysing the information gathered, Brazil does not have an adequate legal basis to guide the environmental remediation of uranium milling and mining facilities. Furthermore, it's necessary that Brazil establishes a specific national policy, that promotes it's objectives clearly and transparently throughout all the life cycle of environmental remediation projects.
\end{abstract}

Keywords: Decommissioning; Legislation; Minning.

\section{Resumen}

La remediación ambiental y el desmantelamiento son tendencias de desarrollo tecnológico que merecen especial atención. Este artículo tiene como objetivo identificar las barreras que pueden retrasar el desmantelamiento y la remediación ambiental de las instalaciones de extracción y procesamiento de uranio, identificando posibles soluciones. Así, se realizó una búsqueda en sitios web y bibliografías específicas, con la orientación del apartado 3 del Proyecto CIDER. Al analizar la información recopilada, Brasil no cuenta con una base legal adecuada para orientar la remediación ambiental de las instalaciones de extracción y procesamiento de uranio. Por lo tanto, es necesario que Brasil establezca una política nacional específica, que promueva sus objetivos de manera clara y transparente a lo largo del ciclo de vida de los proyectos de remediación ambiental. 
Palabras clave: Desmantelamiento; Legislación; Minería.

\section{Introdução}

A remediação ambiental e o descomissionamento são de certa forma, assuntos bastante atuais e que requerem atenção. Na primeira metade do século XX, quando já havia extração de urânio por todo o mundo, essas seriam palavras desconhecidas e sem importância. Com o passar do tempo, pelo constante crescimento das exigências legais e da preocupação ambiental, tomou-se partida por diversas frentes o trato desse assunto, com estudos e cobrança às mineradoras espalhadas pelo Brasil e pelo mundo.

Descomissionar, de acordo com a Agência Internacional de Energia Atômica (IAEA, 2016), significa "tomar todas as providências necessárias para a desativação de uma instalação nuclear ao final de sua vida útil, observando-se todos os cuidados para proteger a saúde e a segurança dos trabalhadores e das pessoas em geral, e ao mesmo tempo, o meio ambiente". Já a remediação ambiental é um conjunto de práticas adotadas quando procura-se mitigar ou anular danos ambientais provenientes de uma dada atividade antrópica.

Em 1951, com a criação do Conselho Nacional de Pesquisa (CNPq), pela Lei 1.310 (Brasil, 1951), foi dado um importante passo para a organização e controle do setor nuclear brasileiro. Com a criação da Comissão Nacional de Energia Nuclear (CNEN), em 10 de outubro de 1956, pelo Decreto 40.110 (Brasil, 1956) subordinada diretamente à Presidência da República, como órgão de política atômica em todos os âmbitos, foram estabelecidos aspectos importantes para o gerenciamento dessa atividade de mineração no Brasil, evidenciando sua importância e a segurança necessária durante a exploração. Com o passar dos anos e diversos Decretos e Leis criadas, a segurança nuclear é baseada em modelos de outros países - principalmente a França - e a CNEN. Segundo Lainetti (2008), a CNEN é o órgão regulatório responsável pela regulamentação, licenciamento e controle da utilização da energia nuclear, pela pesquisa e desenvolvimento, pela produção de radioisótopos e deposição do rejeito radiativo no Brasil.

De acordo com Cipriani (2002), o Complexo Mínero-Industrial do Planalto de Poços de Caldas (CIPC) é o primeiro empreendimento de lavra e tratamento de minério de urânio a operar no Brasil. A produção industrial de urânio teve início em 1982 no CIPC, uma instalação com mina e usina de tratamento químico, onde foram produzidas 1.200 toneladas de urânio (tU). O CIPC funcionou de modo descontínuo devido a uma série de dificuldades atribuídas a pouco conhecimento acerca das características do minério. Sendo assim, a tomada para a solicitação da licença para o descomissionamento está sendo adiada em função dessas diversas indefinições.

Vale lembrar que a população diretamente atingida pela instalação do empreendimento minerário, precisa ter participação no processo decisório, o que não ocorria nos primeiros empreendimentos deste tipo instalados no Brasil. Silva, Cordeiro, Calazans, Alvarenga e Cordeiro (2018) indicam a necessidade de informação da população quanto a todos os processos que envolvem a atividade minerária, bem como os pontos positivos e negativos para a região onde se insere. Sena e Mont-Mor (2018), advertem que os métodos de quantificação e análise dos impactos muitas vezes fornecem resultados discrepantes em relação à percepção real da população do entorno do empreendimento.

O objetivo deste trabalho foi identificar barreiras que possam impedir o avanço do Descomissionamento e Remediação Ambiental (D\&RA) de instalações de mineração e beneficiamento de urânio no Brasil e identificar possíveis soluções, realizando um levantamento relativo ao descomissionamento e remediação ambiental, analisando os problemas tecnológicos e de infraestrutura e aplicando a Seção 3 do documento do projeto CIDER.

\section{Metodologia}

Foi adotado o método de pesquisa quantitativo (Pereira, Shitsuka, Parreira \& Shitsuka, 2018) que, de acordo com 
Martins (2004), é entendido como o conhecimento crítico dos caminhos do processo científico, indagando e questionando acerca de seus limites e possibilidades, onde toda questão técnica implica uma discussão teórica. Segundo Lakatos e Marconi (2013), constitui o método de abordagem dedutivo.

Foram utilizados dados secundários, levantamento bibliográfico e documental, tendo como base as guide lines da seção 3 do documento CIDER da IAEA, assim como a legislação pertinente e sites de instituições, no que diz respeito à questão da remediação ambiental de empreendimentos de mineração e beneficiamento de urânio no Brasil.

\section{Resultados e Discussão}

\subsection{Descomissionamento e remediação ambiental}

A preocupação com o legado para as gerações futuras tem adquirido cada vez mais importância na avaliação de novos projetos, no funcionamento dos empreendimentos e até no fechamento definitivo dos mesmos. O ciclo de vida de um empreendimento mineral costuma ser caracterizado tradicionalmente pelas fases de exploração mineral, pesquisa da jazida, desenvolvimento da mina, lavra e exaustão da jazida. Durante a produção, o empreendimento pode vir a ser paralisado temporariamente. A exaustão da jazida encerra definitivamente o empreendimento e o local geralmente é abandonado. A paralisação temporária ou o fechamento definitivo da mina acarreta impactos de ordem social e ambiental sobre a região ou país em que ela está localizada.

Para a aprovação de novos projetos, começaram a ser exigidos em alguns países não só um plano de fechamento futuro, como também a alocação de recursos para este fim antes da implantação do empreendimento. Esse processo pode ser chamado de remediação, que visa a remoção da fonte de contaminação, a redução de contaminação do solo e da água subterrânea para níveis aceitáveis ambientalmente, de riscos ambientais ou de exposição de trabalhadores e usuários do local e do recurso. Isso tudo vem atrelado ao descomissionamento, o conjunto de operações necessárias para a perfeita garantia da desativação da mina, visando devolver o local para outros usos pela comunidade.

O Programa Poland and Hungary: Assistance for Restructuring their Economies (PHARE), que inicialmente foi um instrumento da União Europeia no auxílio aos países do Leste Europeu nos preparativos para se integrarem à própria, foi o principal meio para a União Europeia obter cooperação técnica e financeira com os Países Centrais e do Leste Europeu (Central and Eastern European Countries, CEEC). Estes países passaram por uma avaliação minuciosa para medir seu progresso e serem incorporados à União Europeia (Webster \& Vrijen, 2001).

Ainda neste contexto, vários países implementaram projetos de desmantelamento consideráveis e de grande importância, um exemplo é o Programa UMTRA (Ação Remediadora de Rejeitos de Urânio) nos EUA. No âmbito deste programa, muitas tecnologias de reparação foram desenvolvidas e soluções-piloto foram concebidas. Sob o Programa, um total de 24 locais de processamento de urânio, com 5.200 propriedades vizinhas associadas, foram fechados e reabilitados a partir de 1978 (Hagen, 2000).

A intenção principal do UMTRA foi criar uma política ambiental para garantir um meio ambiente seguro e protecionista, tanto para o público quanto para os trabalhadores, através de medidas sustentáveis do dia-a-dia que atendiam às leis, normas e padrões de proteção ambientais. Também visava assegurar a transparência das ações executadas e decisões tomadas na implementação de certos princípios para a utilização correta e ética dos recursos naturais, a condução correta e ambientalmente segura das operações, cumprir obrigações com os órgãos ambientais, prevenir poluição, emissão de resíduos, reciclar onde for possível e continuamente melhorar o sistema de gestão ambiental para maior eficiência.

Os países que enfrentam problemas para descomissionamento e remediação de áreas, desenvolvem tecnologia para caracterizar os rejeitos e assim, poder tratá-los. O custo nesse tratamento pode chegar a ser muito maior que o preço de extração, visto que é necessário um grande período de tempo, estudo e garantias ambientais a serem seguidas. 


\subsection{Descomissionamento e remediação ambiental no Brasil}

Segundo Matos e Rubini (1999), devido às dificuldades de logística e dimensões do território nacional, apenas 1/3 do território nacional foi submetido à pesquisa uranífera. As regiões dos maiores depósitos e ocorrência de urânio no Brasil estão dispostas no Quadro 1.

Quadro 1. Principais depósitos e ocorrências de reservas de urânio no Brasil, em toneladas métricas de U3O8.

\begin{tabular}{|c|c|c|c|c|c|}
\hline \multirow{3}{*}{$\begin{array}{c}\text { JAZIDA/ } \\
\text { DEPÓSITO }\end{array}$} & \multicolumn{4}{|c|}{ CLASSE DE RESERVA } & \multirow{3}{*}{$\begin{array}{l}\text { TOTAL } \\
\text { GERAL }\end{array}$} \\
\hline & \multicolumn{3}{|c|}{ MEDIDA/INDICADA } & \multirow{2}{*}{$\begin{array}{c}\text { INFERIDA } \\
\mathrm{U}_{3} \mathrm{O}_{8}<40 \mathrm{US} \$ / \mathrm{lb}\end{array}$} & \\
\hline & $\mathrm{U}_{3} \mathrm{O}_{8}<20 \mathrm{US} \$ / \mathrm{lb}$ & $\mathrm{U}_{3} \mathrm{O}_{8}<40 \mathrm{US} \$ / \mathrm{lb}$ & TOTAL & & \\
\hline CIPC & - & 500 & 500 & 4.000 & 4.500 \\
\hline LAGOA REAL & 24.200 & 69.800 & 94.000 & 6.770 & 100.770 \\
\hline ITATAIA & 42.000 & 41.000 & 83.000 & 59.500 & 142.500 \\
\hline OUTRAS $^{b}$ & - & - & - & 61.600 & 61.600 \\
\hline TOTAL & 66.200 & 111.300 & 177.500 & 131.870 & 309.370 \\
\hline
\end{tabular}

Fonte: Matos e Rubini (1999).

Atualmente, é evidenciada a preocupação com o descomissionamento e a remediação de instalações de mineração e beneficiamento de urânio. Cipriani (2002), em seu estudo na Unidade de Tratamento de Minério (UTM) - INB Caldas, debateu diversos aspectos a respeito desse assunto no cenário brasileiro. Enfatizou que a Constituição Federal de 1988 (Brasil, 1988) obriga o minerador a recuperar o meio ambiente degradado pela atividade mineradora, de acordo com a solução técnica do órgão competente, seguindo normas ambientais.

De acordo com Fernandes (2010), as mudanças de comportamento e procedimento foram fruto da conjunção da nova visão política de proteção ambiental e do conceito de desenvolvimento sustentável. A reação da sociedade contra as condições de abandono das minas e usinas de urânio no Brasil deu lugar a inúmeras audiências públicas, que levaram a inquéritos, implantados pelo Poder Judiciário, para investigação dos possíveis impactos dos rejeitos das usinas de urânio sobre o meio ambiente e a saúde da população.

\subsection{Descomissionamento e remediação ambiental no Planalto de Poços de Caldas}

Segundo Fernandes (1997; 2010), a produção industrial de urânio no Brasil começou no Complexo Mínero-Industrial do Planalto de Poços de Caldas, em 1982, 30 anos depois das primeiras pesquisas desse bem mineral no país.

Para manter a licença de operação, a empresa deve possuir um Serviço de Radioproteção e desenvolver planos de monitoração, controle e operação. Esses planos, em função do desenvolvimento da operação e de necessidades de alteração do processo são revisados e submetidos à CNEN. Em cumprimento ao Plano de Monitoração Ambiental (PMA) da instalação, o Serviço de Radioproteção executa medidas de radiação gama externa, de concentração de atividade de radionuclídeos específicos em amostras de águas de superfície e subterrânea, ar, aerossol, solo, sedimentos, produtos agropecuários (legumes, verduras, grãos, frutas, carne, leite, ovos) peixes e pasto dentro das próprias instalações e até um raio de $30 \mathrm{~km}$, atingindo cidades próximas (Cipriani, 2002).

Atualmente, as Indústrias Nucleares do Brasil (INB), unidade Caldas, se dedica aos bota-foras e à mina, tratando a drenagem ácida gerada. Porém todas as ações carecem de um plano juntamente com a CNEN e o IBAMA, que possuem competência legal para fiscalizar.

Trabalhos relacionados ao conteúdo de urânio em materiais biológicos nesta região foram produzidos, como o estudo de Pereira, Espindola, Silva e Kelecom (2015), demonstrando a necessidade de desenvolvimento de técnicas mais apuradas para deteç̧ão, e consequentemente adequado monitoramento ambiental, nesta e em outras regiões. 


\subsection{Projeto CIDER (IAEA, 2016)}

$\mathrm{O}$ documento do projeto CIDER (Constraints to Implementation of Decommissioning and Environmental Remediation) é composto por 6 seções, divididas em subseções, contendo informações relevantes a respeito de aspectos técnicos, políticos, financeiros e socioculturais, com o intuito de elevar os níveis de performance de projetos de descomissionamento e remediação ambiental de instalações nucleares.

\subsubsection{Política Nacional}

A política nacional para o D\&RA define as principais responsabilidades para a implementação do programa, seja pelo governo ou empresas privadas, fazendo preparativos para arrecadação de fundos.

Autoridades legais também necessitam tomar precauções para assegurar uma ampla participação de stakeholders, incluindo o público em geral e especialmente comunidades locais, buscando implementar discussões que são abertas e inclusivas, que aceitas por todos.

A política desenvolvida deve procurar seguir os princípios:

I. Promover proteção das pessoas e do meio ambiente, tanto agora quanto no futuro;

II. Incluir um compromisso a longo termo para garantir que sites e resíduos serão propriamente gerenciados;

III. Promover continuidade e eficiência na utilização de recursos humanos e financeiros;

IV. Promover interações abertas e transparentes com os stakeholders.

Além disso, políticas nacionais para sítios contaminados devem refletir a magnitude e escala do perigo em potencial, devendo ser interligada com as políticas de gerenciamento de resíduos existentes. A política nacional deve refletir prioridades nacionais, circunstanciais, a disposição dos recursos humanos e financeiros, podendo ser influenciada por diversos fatores, como o momento escolhido para a remediação ambiental.

A ausência de uma política nacional pode resultar em obstrução do órgão regulatório, atrasando o processo de D\&RA. Pode também resultar em falta de motivação nos responsáveis pelo sítio em iniciar o processo. Se a política não é suficiente ou é incompleta, pode-se perder o foco dos projetos de D\&RA, causando uso ineficiente de recursos, resultando em uma grande chance de tais projetos serem abandonados.

\subsubsection{Estrutura legal e regulatória}

A estrutura legal e regulatória compreende um grupo de arranjos que estabelecem o processo regulatório. Determina um ou mais órgãos regulatórios a desenvolver normas condizentes com as atividades do D\&RA e fiscalizar tais atividades com base nestas normas. É necessário que esteja alinhada com outros elementos da política nacional. A estrutura deve ser dedicada aos projetos ou embutida em leis genéricas relacionadas à energia nuclear.

Requisitos regulatórios devem ser extremamente detalhados e específicos (lê-se regulação prescritiva) ou podem ser mais gerais e baseadas em objetivos, permitindo aos operadores oportunidades de proporem abordagens detalhadas para seu cumprimento (lê-se regulação baseada em performance). Podem existir diferenças significantes nas abordagens dependendo no sistema nacional regulatório ou práticas comuns baseadas em tradições culturais. Entretanto, quaisquer abordagens precisam ter a escala apropriada aos projetos de D\&RA, levando em conta os maiores requisitos de segurança onde existam maiores riscos à segurança.

Os problemas a seguir devem ser abordados durante o desenvolvimento da estrutura legal para D\&RA:

I. Justificar o conteúdo da estrutura em termos de risco e custo benefício;

II. Verificar o cumprimento de recomendações internacionais estabelecidas em tratados ou convenções relevantes;

III. Demonstrar que a estrutura reflita boas práticas, buscando sempre o engajamento de stakeholders em decisões 
ambientais e quaisquer outras que possam ser implementadas para um controle adequado;

IV. Obter uma aprovação prévia dos órgãos regulatórios no caso de atividades que possam representar um risco à segurança de indivíduos ou ao meio ambiente, como radiação e tecnologia baseada em energia nuclear.

A estrutura legal e regulatória precisa ser apropriada para o alcance de instalações e atividades relevantes. O resultado do documento CIDER indicou que, em determinadas circunstâncias, a estrutura legal e regulatória para D\&RA é completamente ou parcialmente inexistente (ou sem relevância). Um possível conflito entre órgãos regulatórios também pode existir. A instituição responsável por conduzir ou regular o projeto de D\&RA pode não estar bem definida. Cada um destes problemas, podem atrasar e dificultar a implementação do projeto.

As principais funções do(s) órgão(s) regulatórios(s) são, resumidamente, garantir a segurança e proteção ambiental. Na prática, são responsáveis por:

\section{Estabelecer regulamentações para D\&RA; \\ II. Garantir o comprimento de normas e leis; \\ III. Rever e aprovar programas de D\&RA;}

IV. Questionar autorizações ou licenças para D\&RA;

V. Avaliar programas de monitoramento durante e depois do D\&RA;

VI. Aprovar mecanismos de controle para utilização futura da área;

VII. Rever e aprovar mudanças em procedimentos ou métodos;

VIII. Avaliar relatórios de ocorrências anormais;

IX. Conduzir inspeções e conduzir ações de correção onde for necessário.

O cumprimento destes papéis e responsabilidades garante a independência do órgão regulatório de organizações e atividades sendo reguladas. Um processo regulatório ineficaz pode ser resultado de uma deficiência de execução e organização dentro do órgão regulatório, advinda, por exemplo, de falta de liderança ou um gerenciamento inadequado, poucos recursos, falta de pessoal ou suporte técnico.

Onde o órgão regulatório não está devidamente organizado e posicionado, os programas de D\&RA podem carecer de direcionamento, conduzindo a falhas. Se o órgão regulatório não é adequadamente independente da organização que implementa o programa, o mesmo pode enfrentar dificuldades associadas com baixa confiança pública, pouca transparência, inconsistência quando comparada com outras iniciativas. Como resultado, o plano pode não ser cumprido ou sequer existir.

Regulamentações nacionais e guias associados ao D\&RA tem a intenção de garantir a segurança da mão de obra, do público e do meio ambiente durante e depois da implementação dos projetos. Seu conteúdo deve ser consoante com o sistema legal nacional, juntamente com padrões internacionais, e devem refletir uma prática comprovada. Requerimentos regulatórios devem refletir uma abordagem graduada em paralelo com a escala do D\&RA previsto.

Durante o desenvolvimento de leis e normas, é importante mensurar seus impactos para promover confiança na eficiência e efetividade das normas propostas. Interações públicas devem ser levadas em consideração durante seu desenvolvimento para prover oportunidades aos stakeholders em opinar antes que sejam finalizados.

\subsection{Análise dos problemas tecnológicos e de infraestrutura}

Com relação aos problemas tecnológicos e de infraestrutura, estes são relatados desde o início da extração de urânio no mundo. Com o passar do tempo e com a preocupação ambiental em evidência, todas as mineradoras começaram a investir e ir em busca de soluções nesse sentido. No Leste Europeu, no início da extração de Urânio, pouco se falava nesse sentido de remediação e descomissionamento das localidades mineradas, tornando o espaço após seu uso sem nenhuma função, acarretando sérios problemas ambientais. 
Essa análise dos países em que houve desenvolvimento da indústria de urânio permite verificar que, de início, devido à falta de legislação adequada, conhecimento e vontade política, as medidas tomadas foram simplistas e acarretaram significativos impactos ambientais e sociais que só começaram a serem mitigados quando a sociedade tomou consciência dos possíveis danos que isso poderia acarretar (Fernandes, 2010).

No Brasil, atualmente busca-se levar em conta um bom investimento inicial para o trabalho de remediação na mina após a mineração completa. Os estudos na área avançam financiados pelas próprias mineradoras, universidades e iniciativas privadas, que visam resultados satisfatórios nesse aspecto, para cada vez mais encontrar bons resultados no âmbito ambiental (Fernandes, 2010). Santos (2008) relata um caso de descomissionamento de uma usina de produção de hexafloreto de urânio (UF6), na qual foi realizada a remoção completa de todos materiais e equipamentos, tratados e/ou armazenados em instalações adequadas.

\subsection{Aplicação da seção 3 do documento do projeto CIDER}

A seção 3.1 do projeto CIDER (IAEA, 2016) afirma que uma política nacional para descomissionamento e remediação ambiental deve: (i) definir as principais responsabilidades para implementação; (ii) estabelecer mecanismos para acumulação e desembolso de fundos; e (iii) proporcionar a participação das partes interessadas. Deve-se considerar os objetivos de promover (a) a proteção dos seres humanos e do meio ambiente, (b) o compromisso de longo prazo para o gerenciamento das áreas e dos resíduos, (c) continuidade no uso de recursos humanos e financeiros e (d) interação aberta e transparente com as partes interessadas. $\mathrm{O}$ texto aponta que quando a política nacional é ausente, a autoridade reguladora pode não ter um mandato claro e que além disso, pode faltar motivação entre os proprietários para iniciar a remediação ambiental.

No caso de política nacional debilitada ou ausente, um pré-requisito para colocar as medidas em prática é entender as responsabilidades relevantes e os riscos apresentados ao público e ao meio ambiente. A revisão para políticas incompletas ou ineficazes, precisa ser realizada para apresentar os reais riscos ao público e meio ambiente, tomando por base as revisões fornecidas através das organizações internacionais. Países comprometidos com o estabelecimento da infraestrutura de segurança e organização para um programa nuclear, devem se basear em atribuições das organizações relevantes envolvidas no desenvolvimento de normas para o descomissionamento e infraestrutura da mineração, em âmbitos globais.

A seção 3.2 do projeto CIDER (IAEA, 2016) afirma que a estrutura legal para a remediação ambiental e descomissionamento pode ser acrescentada à legislação nuclear existente, (i) sendo um conjunto de dispositivos legais que estabelecem e sustentam o processo regulatório, (ii) definindo que um ou mais órgãos reguladores desenvolvam normas adequadas, e que naturalmente regulem as atividades de acordo com suas normas. Ainda na mesma seção, o texto afirma que a estrutura regulatória pode ser (i) detalhada e específica (prescritiva) ou (ii) genérica e baseada em metas. Mais adiante, o texto informa que caso não existam estruturas legais e regulatórias adequadas, pode haver conflito entre diferentes órgãos reguladores. Além disso, a ausência de normas específicas pode suscitar projetos mal planejados ou mal gerenciados, frente a uma abordagem regulatória inconsistente.

Para o descomissionamento ser eficaz, o governo precisa estabelecer uma política nacional que seja apoiada por um quadro jurídico e regulamentar. A estrutura precisa identificar a autoridade reguladora e designar poderes e recursos necessários. A autoridade reguladora é mais eficaz quando é independente do operador; isso proporciona responsabilidade, confiança e transparência ao público. Nos casos em que as políticas de remediação ou o quadro legal e regulamentar é incompleto, restringe-se a influência que a autoridade reguladora tem sobre o operador relativo à implementação do descomissionamento (IAEA, 2016).

Todas as medidas estudadas, juntamente com as regulamentações nacionais, destinam-se a garantir a segurança da força de trabalho, da população em geral e do meio ambiente, durante e após a implementação dos projetos. As normas 
seguidas devem ser de acordo com o sistema legislativo nacional, juntamente com as normas internacionais, e devem refletir na prática. Esses requisitos regulamentadores devem evidenciar uma abordagem gradual em consonância com a escala de implementação do projeto, havendo maiores preocupações e responsabilidades onde existem riscos substanciais associados ao projeto.

Em 2018 foi sancionado o Decreto n 9.600 (Brasil, 2018) que trata da consolidação das diretrizes da política nuclear brasileira (Brasil, 2018). Os artigos 13 e 14 do decreto citado tratam dos rejeitos e combustível nuclear utilizado, porém não trazem contribuição adicional à questão do descomissionamento, considerando o já especificado na lei $\mathrm{n}^{\circ} 10.308$ de 2001 (Brasil, 2001).

\section{Considerações Finais}

O desenvolvimento do presente estudo possibilitou uma análise a respeito das barreiras da implementação da remediação no Brasil e seus problemas, quando não existem estruturas legislativas adequadas. Quando analisado em âmbito global, o descomissionamento é considerado um assunto novo e que teve sua relevância reconhecida nos últimos 50 anos.

Dada à importância do assunto, reunir informações sobre o planejamento da mineração de urânio no Brasil é muito válido, devido aos riscos ambientais e sociais relacionados ao assunto, possibilitando identificar as principais barreiras. De certa forma, o campo de estudos nessa área ser recente auxilia a traçar novos rumos ao assunto, considerando sua devida importância.

Em relação ao projeto CIDER e seus parâmetros, conclui-se que quando definidas as principais responsabilidades para implementação e estabelecidos os mecanismos para acumulação e distribuição de fundos, assim como a participação das partes interessadas, os empreendimentos podem adequar-se às legislações vigentes, aumentando a probabilidade de se alcançar resultado satisfatório na remediação e no descomissionamento.

O Brasil ainda não dispõe de uma política nacional ou de uma base legal adequada para guiar a remediação ambiental de empreendimentos de mineração e beneficiamento de urânio. Finalmente, a sugestão é que se estabeleça uma política nacional para esta finalidade, passando pela concepção da base legal necessária, culminando na publicação de normas específicas que promovam clareza e transparência ao longo de todo o ciclo de vida dos projetos de remediação.

Para trabalhos futuros, sugerimos o acompanhamento da evolução das normativas legais e estudo da aplicação efetiva das mesmas nos processos de descomissionamento referentes à mineração e beneficiamento de urânio.

\section{Agradecimentos}

Ao Laboratório de Poços de Caldas (LAPOC) da Comissão Nacional de Energia Nuclear (CNEN); ao Programa de Pós-Graduação em Ciências Ambientais da Universidade Federal de Alfenas (PPGCA/UNIFAL) e à Coordenação de Aperfeiçoamento de Pessoal de Nível Superior - Brasil (CAPES).

\section{Referências}

Lei $n^{\circ}$ 1.310, de 15 de janeiro de 1951. Cria o Conselho Nacional de Pesquisas e dá outras providências. https://www2.camara.leg.br/legin/fed/lei/19501959/lei-1310-15-janeiro-1951-361842-normaatualizada-pl.pdf

Decreto $n^{o}$ 40.110, de 10 de outubro de 1956. Cria a Comissão Nacional de Energia Nuclear e dá outras Providências. Recuperado de: http://legis.senado.gov.br/legislacao/ListaTextoSigen. action?norma=462693\&id=14232413\&idBinario=15772511\&mime=application/rtf

Constituição da República Federativa do Brasil. (1988). Brasília, DF: Senado Federal: Centro Gráfico, 292 p.

Lei $n^{\circ}$ 10.308, de 20 de novembro de 2001. Dispõe sobre a seleção de locais, a construção, o licenciamento, a operação, a fiscalização, os custos, a indenização, a responsabilidade civil e as garantias referentes aos depósitos de rejeitos radioativos, e dá outras providências. Recuperado de: http://www.planalto.gov.br/ccivil_03/leis/LEIS_2001/L10308.htm 
Research, Society and Development, v. 10, n. 1, e19310110806, 2021

(CC BY 4.0) | ISSN 2525-3409 | DOI: http://dx.doi.org/10.33448/rsd-v10i1.10806

Decreto $n^{\circ}$ 9.600, de 5 de dezembro de 2018. Consolida as diretrizes sobre a Política Nuclear Brasileira. Recuperado de: https://www.in.gov.br/materia//asset_publisher/Kujrw0TZC2Mb/content/id/53757734/do1-2018-12-06-decreto-n-9-600-de-5-de-dezembro-de-2018-53757633

Cipriani, M. (2002). Mitigação dos impactos sociais e ambientais decorrentes do fechamento definitivo de minas de urânio. . http://bdtd.ibict.br/vufind/Record/CAMP_ee8167eec65049d6fa0b6cbcde788562

Fernandes, H. M. (1997). Subsídios ao descomissionamento da primeira indústria de mineração e beneficiamento de urânio no Brasil. O caso do Complexo Mínero Industrial de Poços de Caldas. Tese (doutorado em Geoquímica), Departamento de Geoquímica da Universidade Federal Fluminense. Niterói. 250 p.

Fernandes, H. M. (2010) Identifying and overcoming the constraints that prevent the full implementation of decommissioning and remediation programs in uranium mining sites. 7 f. Journal of Environmental Radioactivity (Institute of Radiation Protection and Dosimetry) - Brazilian Nuclear Energy Commission, Rio de Janeiro.

Hagen, M. (2005). Decommissioning and rehabilitation of uranium and thorium production facilities. $91-99$ f. Kerntechnik. https://portale.wisutec.de/Portals/9/publication/Kerntechnik_2005-U-Th-ecommissioning.pdf

IAEA. International Atomic Energy Agency. (2016). Advancing implementation of decommissioning and environmental remediation programmes: CIDER project: baseline report. IAEA Nuclear Energy Series No. NW-T-1.10, Viena. https://www-pub.iaea.org/books/iaeabooks/10993/Advancing-Implementationof-Decommissioning-and-Environmental-Remediation-Programmes

Lakatos, E. M., \& Marconi, M. de A. (2003). Fundamentos de metodologia científica. (5a ed.), Atlas.

Lainetti, P. E. de O. (2008). Desmantelamento e descomissionamento de instalações nucleares no Brasil. Instituto de Pesquisas Energéticas e Nucleares IPEN/CNEN-SP. Comissão Nacional de Energia Nuclear. https://www.ipen.br/biblioteca/2008/eventos/13601.pdf

Martins, H. T. S. (2004). Metodologia qualitativa de pesquisa. Educ. Pesqui. 30(2), 289-300. 10.1590/S1517-97022004000200007

Matos, E. C., \& Rubini, L. A. (1999). Reservas brasileiras de urânio e sua capacidade de atendimento à demanda interna. Resende: Indústrias Nucleares do Brasil S.A. - INB. 7 p.

Pereira, W. S.; Espindola, C. B.; Silva, A. X., \& Kelecom, A. (2015). Urânio em material biológico III. Urânio em Minas Gerais, Brasil. Anais... X Congreso Regional Latinoamericano IRPA de Protección y Seguridad Radiológica "Radioprotección: Nuevos Desafíos para un Mundo en Evolución". Buenos Aires, 12 al 17 de abril. Sociedad Argentina de Radioprotección. http://www.irpabuenosaires2015.org/Archivos/trcompletos/irpa/5_pereira_etal_IRPA_U_MB_UTM.pdf

Pereira A. S., Shitsuka, D. M., Parreira, F. J. \& Shitsuka, R. (2018). Metodologia da pesquisa científica. [e-book]. 1 Ed. Santa Maria, RS: UFSM, NTE. https://repositorio.ufsm.br/bitstream/handle/1/15824/Lic_Computacao_Metodologia-Pesquisa-Cientifica.pdf?sequence=1

Santos, I. (2008). Descomissionamento de uma usina de produção de hexafluoreto de urânio. Tese. Doutorado em Ciências na Área de Tecnologia Nuclear Materiais. Instituto de Pesquisas Energétics e Nucleares. Universidade de São Paulo. http://pelicano.ipen.br/PosG30/TextoCompleto/Ivan\%20Santos_D.pdf

Sena, C. S., \& Monte-Mor, R. C. A. (2018). Nuances da Relação da Percepção Ambiental e os Impactos Ambientais da Mineração em Periquito no Bairro Vila Amélia em Itabira/MG. Research, Society and Development, 7 (11), 01-21, e871146. 10.33448/rsd-v7i11.460

Silva, D. M.; Cordeiro, J.; Calazans, G. M.; Alvarenga, C. A. \& Cordeiro, J. L. (2018). Percepção dos moradores de Barão de Cocais (MG) acerca da criação do Parque Nacional da Serra do Gandarela e dos impactos desencadeados pela atividade minerária. Research, Society and Development, 7(1), 1-20, e371112. doi: $10.17648 /$ rsd-v7i1.94

Webster, S., \& Vrijen, J. (2001). The legacy of uranium mining in Central and Eastern Europe-a view from the European Union. International Agency of Atomic Energy - IAAE. https://inis.iaea.org/collection/NCLCollectionStore/_Public/33/032/33032932.pdf 